\title{
News
}

Viszeralmedizin 29| 2 | 13

Takeda beantragt Genehmigung für das Inverkehrbringen von Vedolizumalb gegen mäßig bis stark alktive Colitis ulcerosa und Morbus Crohn in der Europäischen Union

Takeda Pharmaceutical Company Limited («Takeda») gab Anfang März 2013 bekannt, dass das Unternehmen bei der Europäischen Arzneimittel-Agentur (EMA) einen Zulassungsantrag (MAA, Marketing Authorization Application) für Vedolizumab eingereicht hat, einem experimentellen, darmspezifischen, humanisierten, monoklonalen Antikörper zur Behandlung von Erwachsenen mit mäßig bis stark aktiver Colitis ulcerosa (UC) und Morbus Crohn (MC), den beiden häufigsten Formen chronisch-entzündlicher Darmerkrankungen (CED). Nach Zulassung wäre Vedolizumab das erste und einzige darmspezifische Biologikum gegen UC und MC auf dem Markt.

«Colitis ulcerosa und Morbus Crohn sind chronische, kräftezehrende Erkrankungen, gegen die es so gut wie keine wirksame Therapie gibt. Betroffen sind häufig junge Menschen in ihren besten Jahren», sagte Asit Parikh, M.D., Ph.D.,
Vice President General Medicine bei Takeda (Abb. 1). «Die Ergebnisse von GEMINI, einer Phase-III-Studie zu Vedolizumab, sind vielversprechend. An dieser klinischen Studie nahmen rund 3000 Patienten in nahezu 40 Ländern teil. Damit ist sie die bis heute umfangreichste klinische Studie zu CED.»

Weltweit leiden fast vier Millionen Menschen an CED, davon in der EU mehr als 500000 an UC und ca. 230000 an MC. UC und $\mathrm{MC}$ sind chronische Erkrankungen, die Entzündungen an den Schleimhäuten des Verdauungstrakts hervorrufen. Von Entzündungen bei MC können verschiedene Areale des Verdauungstrakts betroffen sein, UC befällt ausschließlich den Dickdarm. MC und UC können schmerzhaft und kräftezehrend sein; dies führt unter Umständen zu schwerwiegenden Komplikationen und zu beträchtlichen Einschränkungen der Lebensqualität der Patienten.

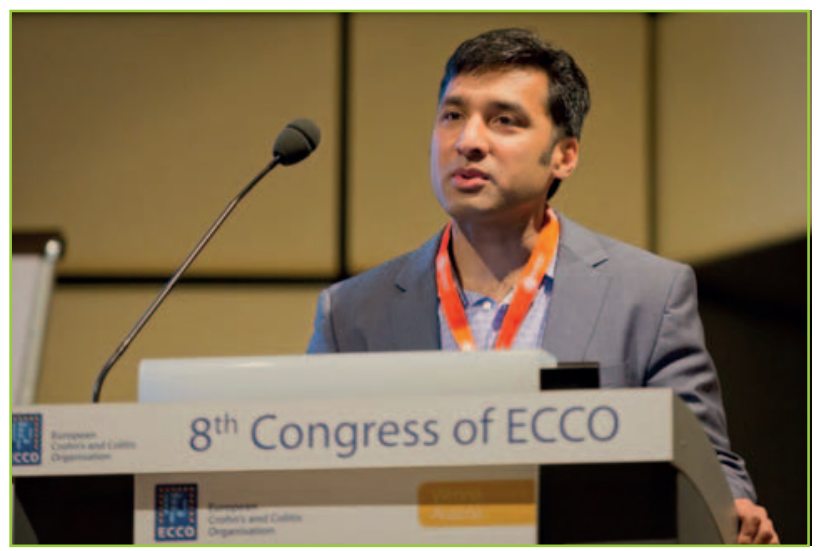

Abb. 1. «UC und $\mathrm{MC}$ sind chronische Erkrankungen, gegen die es so gut wie keine wirksame Therapie gibt. Die Studienergebnisse zu Vedolizumab sind vielversprechend», erläutert Vice President General Medicine M.D., Ph.D. Asit Parikh.
Der Zulassungsantrag stützt sich auf die Phase-III-Studien GEMINI I, GEMINI II, GEMINI III und GEMINI LTS (Long-Term Safety/langfristige Sicherheit); diese sind Teil der GEMINI Studies(TM), einem aus vier Studien bestehenden klinischen Programm zur Untersuchung der Wirksamkeit und Sicherheit von Vedolizumab gemessen an klinischem Ansprechen und Remission bei Patienten mit mäßig bis stark aktiver UC und $\mathrm{MC}$, bei denen zumindest eine herkömmliche oder Anti-TNF-alpha-Therapie erfolglos war.

«Durch den spezifischen Wirkungsmechanismus ist Vedolizumab klinisch vielversprechend als Therapiemöglichkeit für Patienten mit mäßig bis stark aktiver UC und $\mathrm{MC}$ », sagte Professor Paul Rutgeerts von der katholischen Universität Löwen, Belgien. «Es ist keine Therapie bekannt, die diese Erkrankungen heilen kann; es besteht Bedarf an neuen Behandlungsoptionen für $\mathrm{MC}$ und UC, damit Patienten weitere Therapien erhalten, die die Symptome abschwächen und Remissionen ermöglichen.»

Weitere Informationen bei

Ketchum Pleon GmbH

Nicole Kromayer

Theresienhöhe 12, 80339 München

Tel. +49 89 59042-1207, Fax -238

Nicole.Kromayer@ketchumpleon.com

\section{Ticker+++ Ticker+++ Ticker+++ Ticker+++ Ticker+++ Ticker+++ Ticker+++}

Bayer. Beim diesjährigen ASCO-GI-Kongress wurden neue Daten zum Krebsmedikament Regorafenib vorgestellt. Darunter sind neue Analysen aus der zulassungsrelevanten Phase-III-Studie CORRECT mit Regorafenib bei metastasiertem Darmkrebs sowie Ergebnisse einer Phase-I-Studie von Regorafenib bei hepatozellulärem Karzinom. Regorafenib wurde im September 2012 auf Basis der CORRECT-Ergebnisse von der Gesundheitsbehörde FDA (USA) unter dem Handelsnamen Stivarga ${ }^{\circledR}$ zur Behandlung von $\mathrm{mCRC}$ zugelassen.

Bayer Pharma AG

Anna Koch

anna.koch@bayer.com
Humedics. Der Spezialist für die akute und mobile Messung der individuellen Leberfunktion am Patientenbett hat eine klinische Phase-IIIStudie gestartet. Das Hauptziel der Studie besteht darin, mithilfe des LiMAx-Tests schneller risikoarme Patienten nach einer Teilentfernung der Leber zu identifizieren und dadurch das Patientenmanagement zu verbessern. Nach Initiierung der ersten Studienzentren sind bereits erste Patienten in die Studie aufgenommen worden.

\section{Humedics GmbH}

Erwin de Buijzer

info@ humedics.de
Ziehm Imaging. Es gibt eine neue Generation mobiler C-Bögen von Ziehm Imaging: Die Ziehm Vision Familie kommt mit optimiertem Design, innovativen Funktionen und SmartDose Dosisreduzierung für den vielseitigen Einsatz bis hin zur minimalinvasiven Gefäßchirurgie. Mit SmartDose sind bei optimaler intraoperativer Bildgebung bereits durch den Einsatz hardwarebasierter Filter und Algorithmen bis zu 20\% Dosiseinsparung möglich.

Ziehm Imaging

Martin Herzmann

Martin.Herzmann@ziehm-eu.com 


\section{News}

Viszeralmedizin 29 | 2 | 13

Palliative $m C R C$-KRAS-wt-Patienten:

\section{Monate medianes Gesamtüberleben dank intensiver} Erstlinientherapie mit Cetuximab + FOLFIRI

Bei Patienten mit metastasiertem Kolorektalkarzinom (mCRC) und KRAS-Wildtyp kann eine intensive Erstlinientherapie bestehend aus Standardchemotherapie und dem EGFR-Antikörper Cetuximab Vorteile bringen. Prof. Dirk Arnold, Freiburg, erläuterte auf der DGHO-Jahrestagung im Oktober 2012 den Stellenwert der Therapieoption unter anderem anhand der Erbitux ${ }^{\circledR}$ Zulassungsstudie CRYSTAL: Insgesamt 1198 therapienaive Patienten erhielten im Verhältnis 1:1 randomisiert entweder eine Behandlung mit einer Standardchemotherapie (FOLFIRI) oder eine Kombination mit Cetuximab. Im Vergleich zur alleinigen Chemotherapie ermöglichte die Zugabe des Antikörpers in der retrospektiven Analyse der 666 KRAS-wt-Patienten eine signifikante Verbesserung im Gesamtanspre- chen (ORR: 57,3 vs. $39,7 \%$; $p<0,001$ ) und eine signifikante Verbesserung des medianen Gesamtüberlebens (OS: 23,5 vs. 20,0 Monate; $p=0,0093$ ). Die Tumorreduktion führte $z u$ einer Verringerung der Symptome und bot die Chance auf Resektion.

Eine retrospektive Subgruppenanalyse der CRYSTAL-Studie zeigte außerdem, dass die Kombination FOLFIRI + Cetuximab eine maximale Symptomlinderung nach bereits 8 Wochen zeigte - im Vergleich zu 16 Wochen bei FOLFIRI alleine. Das Erreichen einer frühen Tumorschrumpfung ( $\geq 20 \%$ innerhalb von 8 Wochen) habe neben dem direkten klinischen Nutzen auch einen hohen prognostischen Stellenwert, so Arnold. Denn im Vergleich zu Patienten ohne frühe Tumorreduktion um $\geq 20 \%$ profitierten Patienten mit früher $\mathrm{Tu}$ morschrumpfung von einem im Durchschnitt 11,4 Monate längeren Gesamtüberleben (30,0 vs. 18,6 Monate; $\mathrm{p}<0,001)$. Fast zwei Drittel $(62 \%)$ der mit der Cetuximab-Kombination behandelten KRAS-wt-Patienten erreichten eine frühe Tumorschrumpfung $\geq 20 \%$, unter FOLFIRI allein war dies nur bei der Hälfte (49\%) der Fall. Der Einsatz des Biomarkers KRAS sowie des klinischen Markers «frühe Tumorschrumpfung» ermöglicht somit eine frühe Prognoseabschätzung.

Literaturangaben und weitere

Informationen bei

Merck Serono GmbH

Yvonne Weißhuhn

Alsfelder Straße 17

64289 Darmstadt

yvonne.weisshuhn@external.merckgroup.com
Adalimumab zur Behandlung des schweren, aktiven pädiatrischen Morbus Crohn zugelassen

Im November 2012 erhielt Adalimumab (Humira ${ }^{\circledR}$ ) die Zulassung zur Behandlung des pädiatrischen Morbus Crohn. Der TNF-Inhibitor ist indiziert zur Behandlung von Kindern und Jugendlichen zwischen 6 und 17 Jahren mit schwerem, aktivem Morbus Crohn, die nur unzureichend auf konventionelle Therapien angesprochen haben, die eine Unverträglichkeit gegenüber einer solchen Therapie haben oder bei denen eine solche Therapie kontraindiziert ist. Damit ist Adalimumab der erste selbst-applizierbare TNF-Inhibitor in dieser Indikation.

Basis der Zulassung sind die Daten der 52-wöchigen, multizentrischen, randomisierten, doppelblinden klinischen Phase-III-Studie IMAgINE 1 [1]. Sie untersuchte körpergewichtsbasiert 2 Dosierungen von Adalimumab hinsichtlich der Re- missionsinduktion und -erhaltung bei 192 pädiatrischen Patienten mit mittelschwerem bis schwerem, aktivem Morbus Crohn, bei denen die konventionelle Behandlung nicht erfolgreich war. Die Patienten waren zwischen 6 und 17 Jahre alt und wiesen einen Pediatric Crohn's Disease Activity Index (PCDAI) Score $>30$ auf. Alle Studienteilnehmer erhielten als Induktionstherapie offen Adalimumab in den Wochen 0 und 2 (160 mg/80 mg bei einem Körpergewicht (KG) $\geq 40 \mathrm{~kg}$ bzw. $80 \mathrm{mg} / 40 \mathrm{mg}$ bei $\mathrm{KG}<40 \mathrm{~kg}$ ). In Woche 4 wurden 188 Patienten nach ihrem Ansprechen und vorheriger Infliximab-Exposition stratifiziert und anschließend randomisiert 2 Adalimumab-Dosierungen zugeteilt. Patienten der Gruppe mit der höheren Adalimumab-Dosis (Standard-Erhaltungsdosis) erhielten über
48 Wochen $40 \mathrm{mg}(\geq 40 \mathrm{~kg} \mathrm{KG})$ bzw. $20 \mathrm{mg}$ ( $<40 \mathrm{~kg} \mathrm{KG)}$ alle 2 Wochen. In der niedrigdosierten Adalimumab-Gruppe wurden $20 \mathrm{mg}$ bzw. $10 \mathrm{mg}$ (je nach $\mathrm{KG} \geq$ oder $<40 \mathrm{~kg}$ ) 14-tägig subkutan als Erhaltungstherapie verabreicht. Zur Woche 26 erreichten 39\% der Patienten mit der Standard-Erhaltungsdosis eine klinische Remission, zur Woche 52 33\% der Patienten mit der Standard-Erhaltungsdosis. Die Sicherheitsdaten der Studie entsprachen dem bekannten Sicherheitsprofil aus anderen Indikationen.

\section{Literatur}

1 Hyams J et al.: Gastroenterology 2012;143(2):365-374.

Weitere Informationen bei

AbbVie Deutschland GmbH \& Co.KG

Maren Nienstedt

Max-Planck-Ring 2a, 65205 Wiesbaden

maren.nienstedt@abbvie.com

\section{Ticker+++ Ticker+++ Ticker+++ Ticker+++ Ticker+++ Ticker+++ Ticker+++}

Roche. Wird der Angiogenese-Hemmer Bevacizumab bei Patienten mit metastasiertem kolorektalem Karzinom ( $\mathrm{mCRC}$ ) kontinuierlich in der Erst- und in der Zweitlinientherapie gegeben, jeweils kombiniert mit einer 5-FU-basierten Chemotherapie, so verlängert dies das Gesamtüberleben signifikant. Der Arzt ist künftig in der Lage, seinen Patienten mit mCRC eine weitere Therapielinie mit Bevacizumab anzubieten.

Roche Pharma AG

Ulla Satzger

ulla.satzger@roche.com
Olympus. Beim 15. Internationalen EndoskopieSymposium in Düsseldorf präsentierten mehr als 30 international renommierte Experten rund 1800 Teilnehmern aus über 50 Ländern die aktuellen Fortschritte in der Endoskopie. Bei der vom Kongresspartner Olympus unterstützten Auftakt-Pressekonferenz stellten Prof. Neuhaus, PD Dr. Schumacher und Prof. Sung die Anwendung neuer Endoskopieverfahren bei Erkrankungen des Verdauungstraktes und die Evaluierung hinsichtlich ihres klinischen Nutzens vor. John Warning Corporate Communications GmbH Martina John m.john@johnwarning.de
Daiichi Sankyo. Der 1. Patient wurde in die weltweite Phase-III-Studie METIV-HCC mit Tivantinib (ARQ 197) eingeschlossen. Tivantinib ist ein in klinischer Prüfung befindlicher selektiver Inhibitor der Rezeptortyrosinkinase c-MET. Die Substanz befindet sich in der letzten Phase der klinischen Entwicklung. Sie wird in der Therapie von Patienten mit hepatozellulärem Karzinom ( $\mathrm{HCC}$ ), die bereits systemisch vorbehandelt sind, untersucht.

Daiichi Sankyo Deutschland GmbH

Pia Nothing

pia.nothing@daiichi-sankyo.de

\section{KARGER}

(1) 2013 S. Karger GmbH, Freibur Verlag, Herausgeber, Redaktion und Verlagsgeschäftsführung übernehmen keine Verantwortung für den Inhalt dieser Rubrik. 